\title{
Using a mechanistic framework to characterise chemistry students' reasoning in written explanations
}

\author{
Patricia Moreira*a, Ainoa Marzabal ${ }^{\mathrm{a}}$, and Vicente Talanquer ${ }^{\mathrm{b}}$ \\ The central goal of this research study was to characterise the different types of reasoning manifested by high school \\ chemistry students when building initial written explanations of a natural phenomenon. In particular, our study participants \\ were asked to explain why a mixture of water and alcohol works as an antifreeze. Data collected in the form of written \\ explanations were analysed using a mechanistic reasoning framework based on the characterisation of system components \\ (e.g..., entities, properties, activities, organisation) and paying attention to the causal models invoked by the participants in \\ their explanations. Our analysis revealed that students at the same educational level construct a wide range of explanations \\ for the same phenomenon that are indicative of different reasoning modes going from descriptive to relational to simple \\ causal to emerging mechanistic. Although the explanations generated by students in our sample were not very sophisticated \\ in terms of the causal models on which they relied, some participants were capable of generating mechanistic explanations \\ using particulate models of matter. The framework for analysis introduced in this contribution can be of use to teachers and \\ researchers in the characterisation of student reasoning
}

\section{Introduction}

As a globalized society we face a variety of social, environmental and technological challenges. To successfully address them, we need to prepare citizens who can understand and reason about the impact that their decisions could have at the personal, community, and planetary levels (Bybee et al., 2009). We require individuals who are able and have the disposition to critically analyse the information they receive, seek scientific explanations, and engage in evidence-based argumentation (National Research Council [NRC], 2013). These abilities require more than a basic understanding of the scientific content typically covered in school science curricula. They demand the integration of central ideas into coherent explanatory frameworks (Millar, 2006). This integration is favoured when students have multiple opportunities to actively engage in building explanations of relevant phenomena (Windschitl et al., 2018). Fostering students' ability to construct scientific explanations is thus seen as critical in the development of functional scientific literacy (Ryder, 2001; Bybee et al., 2009).

The aforementioned educational goals are shared by Chile, the country where the present study was conducted. The Chilean school curriculum highlights the importance of developing students' ability to engage in science practices such as building explanations. However, national and international standardized test results show that the educational system falls

\footnotetext{
a. Facultad de Educación, Pontificia Universidad Católica de Chile, Santiago, Chile. E-mail: pmmoreira@uc.cl.

b. Department of Chemistry and Biochemistry, University of Arizona. Tucson, Arizona 85721, USA.E-mail:vicente@u.arizona.edu
}

short of meeting this goal. According to these data, Chilean students demonstrate low performance in using their scientific knowledge to explain relevant phenomena (Agencia de Calidad de la Educación [ACE], 2015).

Studies about the nature of scientific explanations (Hempel and Oppenheim, 1948; Salmon, 1984; Machamer et al., 2000) and students' ability to generate them (Talanquer, 2010; Braaten and Windschitl, 2011; Rottman and Keil, 2011; Yeo and Gilbert, 2014) are diverse. Some authors have focused on the structure of these explanations (Tang, 2016; Cabello, 2017); others have paid attention to the different functions that explanations serve (Gilbert et al., 2000) or to the types of causal relationships that are established within them (Brewer et al., 1998; Grotzer, 2003; Christidou and Hatzinikita, 2006; Talanquer, 2007, 2010). These different studies emphasize the importance of developing students' explanatory abilities but also reveal the multiple challenges in doing so.

In recent years, there has been increased interest in better characterising and fostering students' ability to generate causal mechanistic explanations (Grotzer, 2003; Russ et al., 2008, 2009; Becker et al., 2016; Southard et al., 2017; Talanquer, 2018). These types of explanations are a hallmark of scientific reasoning and students are expected to generate them using normative concepts and ideas (NRC, 2013). In this paper, we present the results of a study in which different frameworks for the characterisation of mechanistic reasoning were integrated and adapted to analyse students' written explanations in a secondary school chemistry class. Our goal is to illustrate how our approach can be applied to identify and characterise different levels of expressed causal reasoning in students' 
explanations. The proposed analytical framework should be useful to both educational researchers and teachers interested in advancing students' explanatory abilities.

\section{Exploring Causal Reasoning}

Engaging students in explanation creates opportunities for them to organise their ideas and build connections between concepts to make sense of systems and phenomena (Izquierdo and Aliberas, 2004). The specific connections or relationships that students establish between concepts and ideas are indicative of their reasoning sophistication. A variety of authors have explored the nature of these relationships seeking to characterise different types of reasoning (Tamir, 1991; Assaraf and Orion, 2005; Sevian and Talanquer, 2014; Becker et al., 2016; Weinrich and Talanquer, 2016). These studies have shown that student reasoning may range from merely descriptive to mechanistic for individuals at the same educational level.

The level of sophistication or complexity in reasoning has been characterised by analysing the causal models that students deploy along different dimensions, such as the type of agency, the interaction patterns, the level of certainty in the causal connections, and the mechanism that are invoked (Grotzer, 2003; Perkins and Grotzer, 2005). Other authors have considered the number and level of integration of the different elements used in the reasoning process (Biggs and Collis, 1982; Brown et al., 2010). The results of these types of investigations were adapted by Sevian and Talanquer (2014) to define a set of modes of reasoning corresponding to different levels of sophistication in student reasoning in chemistry going from descriptive to relational to linear causal to multi-component. The latter type of reasoning shares many of the characteristics that other authors attribute to mechanistic reasoning.

Mechanistic explanations are characterised by the detailed descriptions of the processes that are responsible for the behaviour of a system of interest (Grotzer, 2003). These types of explanations are based on the identification of relevant entities in a system, their properties and interactions, the activities in which they engage, and the patterns of organisation that they manifest (Machamer et al., 2000). The activities and organisation of core entities are seen as causally responsible for the properties and behaviours of the system as a whole. Interest has grown in recent years in the analysis of mechanistic reasoning in different science fields and the exploration of how to foster its development in science classrooms. Several of these investigations have been carried out in the context of biology education (Machamer et al., 2000; van Mil et al., 2013; Southard et al., 2017), but they have also recently emerged in the field of chemistry (e.g. Bhattacharyya, 2013; Becker et al., 2016; Talanquer, 2018).

Distinguishing mechanistic reasoning from other types of causal reasoning may be challenging. Russ and collaborators (2008) proposed a framework for recognizing student mechanistic reasoning when engaged in scientific inquiry. This framework for discourse analysis was developed by adapting accounts of mechanism from the philosophy of science (Machamer et al., 2000). Within this framework, mechanistic explanations are distinguished from other types of explanations by the description and analysis of the spatio-temporal organisation of the entities that comprise a system. Students are capable of building these types of explanations although the mechanisms that they propose may not align with accepted scientific accounts. The discourse analysis framework for recognizing mechanistic reasoning introduced by Russ and collaborators (2008) includes seven categories of analysis: 1) describing the target phenomenon, 2) identifying setup conditions, 3) entities, 4) activities, 5) properties of entities, 6) organisation of entities and 7) chaining.

In this study, we integrated and adapted different approaches to the characterisation of students' causal reasoning (Grotzer, 2003; Russ et al., 2008; Sevian and Talanquer, 2014) in our analysis of written explanations. Our analytical approach can be used by both teachers and researchers to analyse students' explanations and characterise the level of causal sophistication in their expressed reasoning.

\section{Research Question and Rationale}

This work is part of a larger qualitative research project directed at characterising the types of explanations built by secondary school students. This particular study centred on the analysis of students' explanations of freezing point depression of aqueous solutions and was guided by the following research question:

- What different types of reasoning manifest in students' explanations of the phenomenon under analysis?

The focus on explanations of freezing point depression was partly determined by constraints imposed by the educational setting in which the research took place. Data collection had to be orchestrated with a teacher who was following a pre-set curriculum and had limited flexibility in her schedule for our investigation. Our choice was also influenced by the nature of the scientific explanations often built to make sense of colligative phenomena. These explanations are constructed using a simple particulate model of matter without referring to specific physical or chemical properties of main system components. In these explanations, colligative properties are seen as emerging from changes in the probability of transfer of solvent particles from one phase of matter to another (Talanquer, 2010). Thus, although the invoked model is simple the appropriate mechanistic explanation is quite sophisticated. We hypothesized that these characteristics would increase opportunities to observe a wide range of student explanations. This hypothesis was also informed by existing research on students' difficulties understanding colligative effects. These studies have shown that students often build alternative explanations using an additive versus an emergent reasoning framework (Talanquer, 2010), and that they tend to misinterpret the effect of the solute by assuming that it changes the time it takes for the phase change to occur rather than the temperature of the transition (Çokadar, 2009).

\section{Methodology \\ Context and Participants}

This research was based on the analysis of the explanations generated by 78 students ( 39 Females; 39 Males) enrolled in two $10^{\text {th }}$ grade chemistry classes taught by a teacher who volunteered to 
participate in the study. Study participants attended a Chilean private-subsidized secondary school. Data were collected as part of a lesson on the topic of freezing point depression. The objective of this lesson was to explain the relationship between freezing temperature and solution concentration. All participants gave their consent to participate in the study. As students were underage, consent was also provided by their legal guardians.

\section{Research Instrument}

Our research probe consisted of an in-class activity based on a hypothetical scenario involving the use of automotive antifreeze. The task asked students to explain in writing why an antifreeze mixture had a lower freezing point than the pure solvent. Students were also asked to create drawings to support their explanation, with the expectation that their drawings could provide additional insights into students' thinking about how the phenomenon happened. Research suggests that written responses often focus on dynamic aspects of a phenomenon while pictorial representations emphasize structural features (Akaygun and Jones, 2014). The English version of the task is presented below. Translations from Spanish of this question and associated student responses presented in the Findings section were completed by the third author of this paper who is fluent in both languages. Nuances in the original text may be lost in translation:

Ignacia is about to embark on a trip with her family. They will travel to an area near a mountain range where below $0^{\circ} \mathrm{C}$ temperatures are expected. After packing and loading the car, Ignacia and her family stop by a gas station. The clerk recommends that they take a bottle of antifreeze with them. Ignacia remembers that her chemistry teacher explained that antifreeze is a mixture of water and alcohol used to prevent water from freezing in the car's engine. Inspired by this thought, Ignacia tells her family what her teacher said. Intrigued, Ignacia's brother asks her: why is it that water doesn't freeze when alcohol is added? Place yourself in Ignacia's shoes: how would you explain to your brother why the mixture of water and alcohol works as an antifreeze? (Create a drawing in the provided box to support your explanation).

\section{Data Collection}

Students' explanations were collected at the beginning of the lesson on freezing point depression. This lesson followed a class in which the topic of boiling point elevation had been introduced. The teacher handed a paper copy of the research probe to each student and read it aloud with them. Students were given 10 minutes to complete the assignment. The teacher did not intervene during this time other than to ensure each student completed the task individually. A total of 78 worksheets were collected but only 75 of them included an actual response.

\section{Data analysis}

Students' written explanations and associated drawings were analysed using a constant comparison method looking for emerging patterns in student reasoning (Miles and Huberman, 1994; Creswell, 2013). We adopted a qualitative interpretative perspective (Sandín, 2003) in the characterisation of the different components present in students' explanations and their relationships. To answer our research question, we analysed the data at two different levels. First, we identified core explanatory components and then we sought to make explicit the relationships among them to evaluate the complexity of students' reasoning.

a) Identification of explanatory components: To complete this part of the analysis, we adapted the discourse analysis framework proposed by Russ and collaborators (2008) to characterise students' reasoning. An initial coding system was developed based on the seven categories of analysis suggested by these authors. These categories were applied in the analysis of each student's explanation. The presence of more of these categories in a single explanation was often indicative of a more advanced response. This coding scheme was tested by five different researchers who coded $3 \%$ of the collected explanations. Based on the results of this first analysis, the coding scheme was revised to adjust it to the nature of the collected data. Three of the initial categories (description of target phenomenon, identification of set up conditions, and chaining) were eliminated as they did not help differentiate between explanations or were not significantly present in the explanations generated by our study participants. The revised coding matrix was applied by two of the authors of this study to $40 \%$ of the sample and individual codes were then compared and discussed. Disagreements were resolved through several rounds of discussion which led to adjustments in the coding scheme. The final scheme was applied by the first author of this paper to analyse the remaining explanations in the sample. The scheme included the following categories of analysis:

1. Entities $\left(E_{x}\right)$ : Material components of the system. They may include macroscopic (e.g., substances) or submicroscopic (e.g., particles) components.

2. Properties $(P)$ : Characteristics of the entities that are relevant in building the explanation. They may be general or specific. Some examples include freezing point and mass. The analysis identifies the entity associated with the cited property and the type of property that is invoked.

3. Activities $(A)$ : Actions/interactions cited as causing changes to entities and/or to the whole system. These activities establish explicit cause-effect relationships. The analysis identified what entity is involved in the action or interaction, and, the type of activities (there may be more than one) invoked in the explanation.

4. Organisation $(O)$ : This category refers to the spatialtemporal location of the entities during a determined activity and its causal connection to the properties or behaviour of the system.

5. Representation $(R)$ : This category refers to the extent to which the drawing provides additional insights into student reasoning. Drawings were used in our analysis to identify other explanatory components not present in the written explanations, such as entities or organisation.

b) Identification of relationships among components: Once the different explanatory components in each explanation were 
When water and alcohol are mixed the solution remains in its liquid state when temperatures are lower than the freezing point of the pure solvent (water). This happens because there is a change in the arrangement of the water molecules when alcohol is added to the solution. The alcohol molecules interfere with the organization of the water molecules into a solid form and the effect is larger when more solute is added. Because of this, the temperatures have to be lower than the freezing point of the pure solvent in order for the mixture to freeze. The more solute is present the lower the new freezing temperature will be.

Figure 1: Hypothetical response to our research probe. It corresponds to Level IV (Emerging Mechanistic) in our

identified, their relationships were mapped into a "reasoning diagram." The construction of these diagrams was informed by existing work on causal models and relationships (Grotzer, 2003). These types of diagrams allowed us to more easily differentiate between types of reasoning. This evaluation was guided by the scheme proposed by Sevian and Talanquer (2014) to characterise different modes of reasoning. This scheme was adapted considering the specific nature of our data. In particular, we assigned the different explanations to four different levels: Level I (Descriptive); Level II (Relational); Level III (Simple Causal); and Level IV (Emerging Mechanistic), characterised in detail in the Findings section.

To illustrate our process of data analysis, we present an example of its application using the hypothetical written response included in Figure 1. Our analysis of this explanation in terms of explanatory components reveals references to two

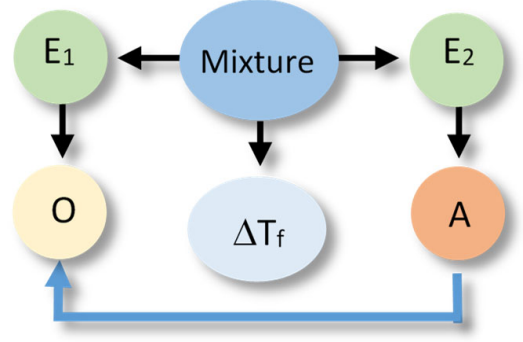

Figure 2. Reasoning diagram corresponding to the explanation in Figure 1. The blue arrow represents the causal relation between the activity (A) of Entity $2\left(E_{2}\right.$, alcohol) and the organization $(O)$ of Entity 1 ( $E_{1}$, water) assumed to cause the change in the freezing point. The symbol $\Delta T_{f}$ represents freezing point depression.

different entities (water particles and alcohol particles) engaged in different activities (e.g., getting arranged, interfering in the arrangement). Additionally, the explanation refers to the organisation of water particles, and the effect of alcohol particles in such organisation, in making sense of the phenomenon observed. No reference to specific properties of the entities in the system is made in the construction of this explanation. The drawing included in Figure 1 is consistent with the associated written response but does not add to its explanatory content. This example is representative of what we observed in the analysis of the explanations of students in our sample. In general, drawings only provided additional insights into student reasoning for some of the explanations at Level IV (Emergent Mechanistic) in our analysis.

The identification of relationships between explanatory components in the hypothetical explanation is summarized in the reasoning diagram in Figure 2 . In this reasoning diagram we make explicit that two entities in the mixture, $E_{1}$ (water) and $E_{2}$ (alcohol) are identified in the explanation. An activity (A) of
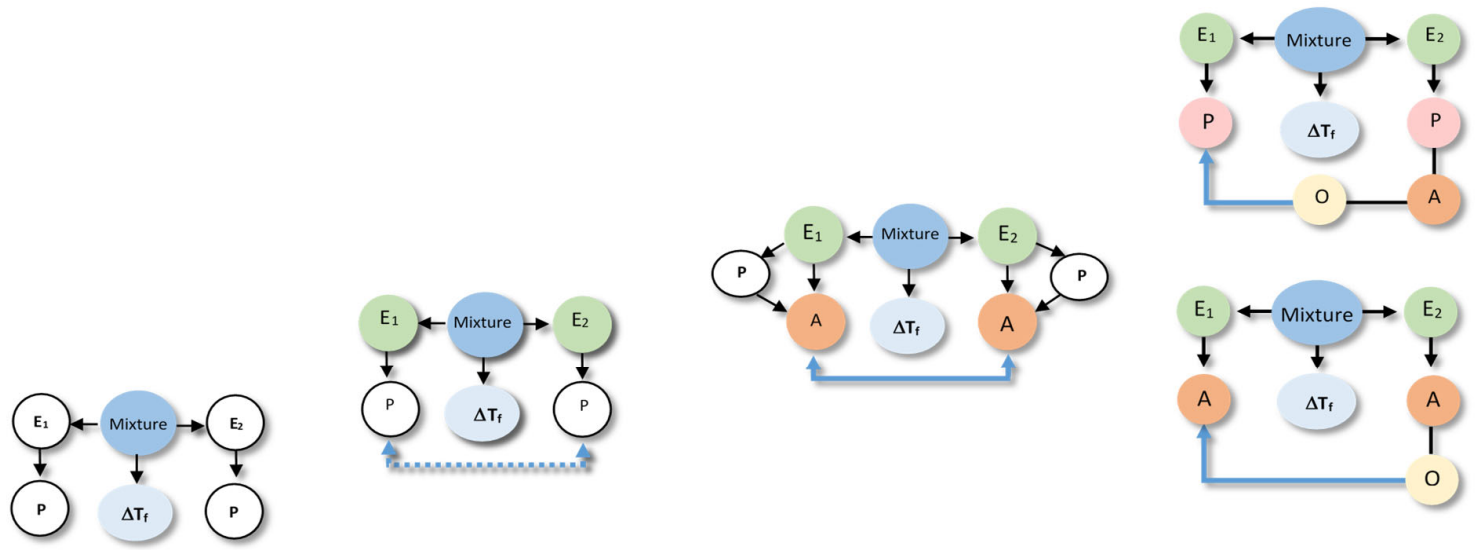

Level I: Descriptive

Level II: Relational

Level III: Simple Causal

Increased Sophistication in Reasoning

Figure 3. Representative reasoning diagrams for the four major levels of reasoning identified in the study. Different colors are used to represent different components in an explanation. Blue dotted arrows in Level II diagrams are used to represent non-causal associations between entities or their properties, while solid blue arrows are used to represent actual causal links. Circles in black and white represent elements that may or may not be present in the explanations at any given level. 
entity $E_{2}$ is assumed to affect the organisation (O) of entity $E_{1}$ as indicated by the direction the arrow from $A$ to $O$.

In the following section we summarize the core findings of our analysis. We only describe patterns of reasoning observed in no less than two students' responses. The described patterns encompass $96 \%$ of the 75 explanations that were analysed.

\section{Main Findings}

The analysis of our study participants' explanations of freezing point depression led us to the identification of 15 different patterns in student reasoning using the analytical approach described in the previous section. Some of these patterns had core characteristics in common that were used to arrange them into four major categories reflecting different levels of sophistication in student reasoning. Representative reasoning diagrams for each of these four categories of reasoning are presented in Figure 3 arranged in order of increased sophistication. Level I (Descriptive) includes responses that are descriptive in nature and limited to restating the phenomenon in different words. Explanations in Level II (Relational) rely on simple associations between properties of the main entities in the system. In Level III (Simple Causal), explanations are based on the activities of one or more of the system's entities, while explanations in Level IV (Emerging Mechanistic) refer to an organisation of entities and activities in space and time to make sense of the phenomenon.

The percentage of explanations in each of the four major levels of reasoning identified in our study is represented in Figure 4 . Close to $15 \%$ of students' responses were at the descriptive level (Level I), while the largest percentage of the explanations (45\%) generated by study participants included simple associations between properties of the main components and the system's behaviour (Level II). Explanations at Level III were found in $28 \%$ of the responses and included reasoning that causally linked the actions of systems' components to the change in the freezing temperature of the solution. Only $12 \%$ of the explanations generated by students were classified at Level IV and referred to some type of mechanism involving the spatio-temporal organisation of

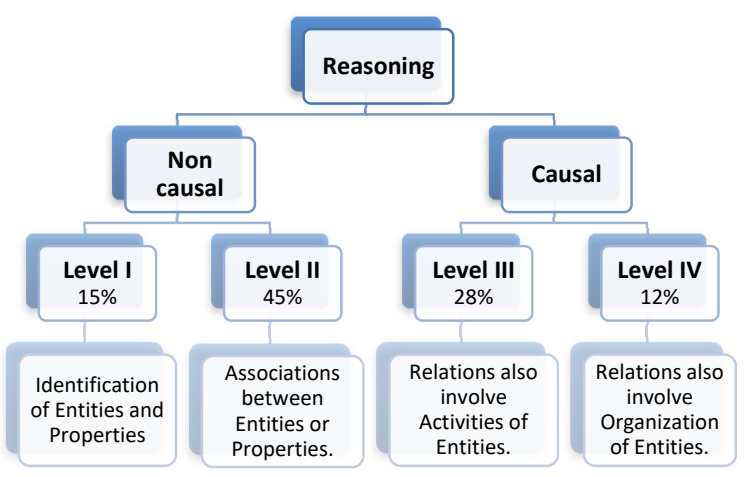

Figure 4: Main types of reasoning manifested in the explanations generated by our study participants.

entities and activities. A more detailed description and analysis of characteristic explanations at each level of reasoning is presented in the following paragraphs.

\section{Level I: Descriptive}

At this first level we placed written answers that were not actual explanations but rather re-descriptions of the phenomenon to be explained (15\%). Responses in this group could include references to the properties of system components (water or alcohol), but without a clear causal link between those properties and the phenomenon under analysis. Examples of responses at this level of sophistication are included in Table 1, together with their associated reasoning diagrams. Responses at Level I were separated into two subgroups based on whether students identified properties of any of the entities in the system. At the L1Sa sublevel, responses referred to the targeted behaviour (anti-freezing) as an intrinsic property of the system (water-alcohol mixture). These types of answers could allude to different system components (see example $i$ in level L1Sa in Table 1) but did not refer to any specific properties of these entities that led to the phenomenon under study. Students'

$\begin{array}{lll}\text { Level } & \text { Reasoning Diagrams and Representative Explanations } \\ \text { L1Sb } & \begin{array}{c}\text { Because water is the solvent and alcohol is the solute. The } \\ \text { reaction causes the solution not to freeze given the } \\ \text { smaller amount of alcohol compared to water (solvent). } \\ \text { 2 students (3\%) }\end{array}\end{array}$

Table 1: Reasoning diagrams and associated representative responses at Level I. 
descriptions at this level were intentional in the sense that alluded to the purpose of the mixture without explaining why the solution worked in such a manner.

Responses at the sublevel $L 1 S b$ involved the recognition of different components present in the system as well as references to properties of those entities (e.g., water easily freezes, alcohol has greater resistance) assumed to be responsible for the observed phenomenon (lower freezing point). However, there was no discussion of how these entities or their properties produced the phenomenon.

\section{Level II: Relational}

This second level of sophistication referred to specific entities, some of their properties, and relationships between them to make sense of the phenomenon. These relationships included unidirectional effects of one entity on another (L2Sa), of the property of one entity over the other entity (L2Sb), of a property of one component over a property of the other ( $\mathrm{L} 2 \mathrm{Sc})$, as well as bidirectional effects between properties of the two components (L2Sd). Specific examples of these different ways of reasoning are included in Table 2 together with their corresponding reasoning diagrams.

In explanations at the sublevel L2Sa, students thought of the alcohol $\left(E_{2}\right)$ as an active agent that caused a change to the water without detailing how the action occurred. At sublevel L2Sb, explanations referred to the properties of one of the entities in the system as responsible for the effect. These types of explanations could refer to a property of the alcohol that interfered with water's freezing process (example $\mathrm{i}$ in L2Sb in Table 2) or to the alcohol directly acting on a property of water (e.g., water's freezing point, as in example ii in L2Sb in Table 2). Similar to explanations in sublevel L2Sa, the relationships invoked were unidirectional with the alcohol acting as the change agent.

Explanations at the sublevels L2SC and L2Sd were characterised by their reference to the properties of both

Level Reasoning Diagrams and Representative Explanations
One of the reasons why it doesn't freeze is because of the
properties of the mixture. It is also because the solvent (water)
is somehow affected by the solute (alcohol), delaying the
freezing process.
2 students (3\%)

L2Sb

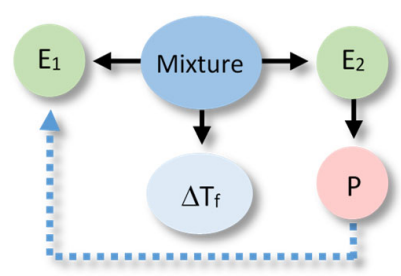

i. $\quad$ Because the alcohol has components that prevent water from freezing.

10 students $(13 \%)$

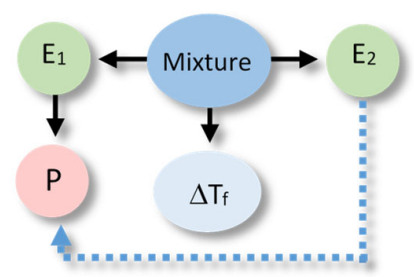

ii. I suppose water only freezes at a certain temperature below $0^{\circ} \mathrm{C}$ and that the anti-freezer with alcohol has a low lower freezing point than pure water. So the alcohol is the solute that in this case affects the water's freezing point. 7 students $(9 \%)$

Ibelieve water doesn't freeze because the alcohol has a lower
freezing point and when mixed with water it changes its
components causing its freezing point to be lower, but not as
much as that of the pure alcohol.

Table 2: Reasoning diagrams and representative responses at Level II. Percentages indicate the relative distribution of explanations (34 total) in this group. Dotted blue arrows are used to represent non-causal associations between entities or their properties. 
entities to explain the observed phenomenon. In the former case (L2Sc), a unidirectional relationship was established with a property of the alcohol (e.g., lower freezing point) affecting a property of water. At the sublevel L2Sd, the properties of both components were seen as responsible for the change in an additive manner (i.e., the properties of the mixture result from the combination of the properties of the components).

\section{Level III: Simple Causal}

Close to $28 \%$ of all explanations were located at the third level of sophistication. These explanations incorporated activities of one or more components of the system as part of their rationale (see Table 3). These activities involved direct action of one entity over the other or were mediated through specific properties of the components. These explanations included more details on the actions of a causal agent and more explicit causal relationships, going from unidirectional relations to cases of mutual causality as illustrated by the sequence of examples in Table 3. Explanations in this category were the most varied in terms of the observed reasoning patterns.

Explanation at the sublevel L3Sa alluded to an action or activity carried out by the alcohol (e.g., increasing the temperature of the system) that affected the other entity. The relationship that was established was unidirectional with the alcohol as the causal agent. At the sublevel L3Sb, alcohol $\left(E_{2}\right)$ was also seen as the active agent affecting the system (e.g., making it warm) through an activity (e.g., particles moving around), but this activity was linked to a property of the entity (e.g., its chemical composition). The explanations located in L3SC share the unidirectional relation with L3Sa and L3Sb. However, L3Sc refers to explanations that invoked activities of

\begin{tabular}{l}
\hline Level \\
L3Sb \\
4 students (5\%)
\end{tabular}

Because alcohol acts as a thermic catalyzer and helps to
vapored water, so not get cold.

L3Se

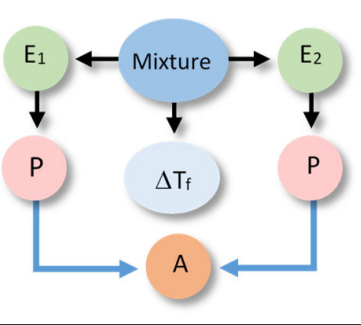

I believe that when water and alcohol interact they produce an effect of more temperature or when both combine they produce more temperature because of the elements in each compound.

4 students $(5 \%)$

Table 3: Reasoning diagrams and associated representative responses at Level III. There were three explanations (4\%) in this group that were unique in terms of their reasoning pattern (not found in more than one explanation). Blue arrows represent major causal links. 
both entities, where the activity of alcohol produced changes in the activity of water.

In explanations at sublevels L3Sd and L3Se, both entities were considered to be engaged in an activity (e.g., producing more temperature, particles moving around) responsible for the phenomenon, without one component being more active than the other. These types of explanations only differed in whether the activity was linked (L3Se) or not (L3Sd) to a specific property of the components.

\section{Level IV: Emerging Mechanistic.}

The most sophisticated types of reasoning were placed in this level (12\%). In these cases, explanations alluded to the spatiotemporal organisation of entities that are involved in activities. Representative examples are included in Table 4.

At the sublevel L4Sa, explanations referred to an activity of alcohol (e.g., acting as a barrier to low temperatures) linked to the spatial organisation of alcohol particles surrounding the water particles. In this case, the organisation of entity E2 affected an E1 activity (e.g., preventing water from freezing). In explanations of type L4Sb, the activity of E1 is linked to a property of this entity (e.g., alcohol has a higher freezing point than water). In both cases, causal relationships are unidirectional with the alcohol acting as the causal agent.

The reference to spatio-temporal organisation of entities in written explanations at Level IV was often complemented by the visual representations present in students' drawings. In particular, students' representations provided additional information about how students thought alcohol acted as a barrier, protecting or hindering water from freezing.

\section{Agency}

Many of the explanations generated by our study participants invoked simple relationships in which an active agent, typically the alcohol through its direct action, due to one of its properties, or by its spatial organisation affected the properties or behaviour of water or the solution. Although causal explanations formally appeared at Level III in our classification, the reference to alcohol as an active agent was present in explanations across Levels II through IV. In these different cases, alcohol was described as taking on one of the four roles described below. In each case, we indicate the percentage of the total responses in the category:

a) Alcohol as a barrier or insulator (20\%): This type of attribution was observed in explanations in Levels II, III and IV. In Level II, students more commonly referred to alcohol as an insulator while in Levels III and IV, the reference to a barrier was more frequent. Explanations at Level IV provided more detailed accounts of how alcohol actually prevented water from freezing. The following excerpts illustrate these different ways of reasoning:

- The alcohol acts as a temperature insulator for water. (L2)

- Because when water is a pure solvent it is more vulnerable to phase changes, because nothing prevents its molecules from ordering. But when adding alcohol, the water molecules are blocked by the alcohol that prevents their phase change. ( L3)

- Maybe because when combining the alcohol with the water it causes the alcohol to be like a barrier, preventing water from freezing because it prevents the below zero temperatures from affecting the water in the car engine. (L4)

b) Alcohol as a source of energy (13\%): This type of agency was found in explanations at Levels II and III. Students at Level III were more likely to refer to the movement of particles as responsible for the observed phenomenon. Consider the following examples:

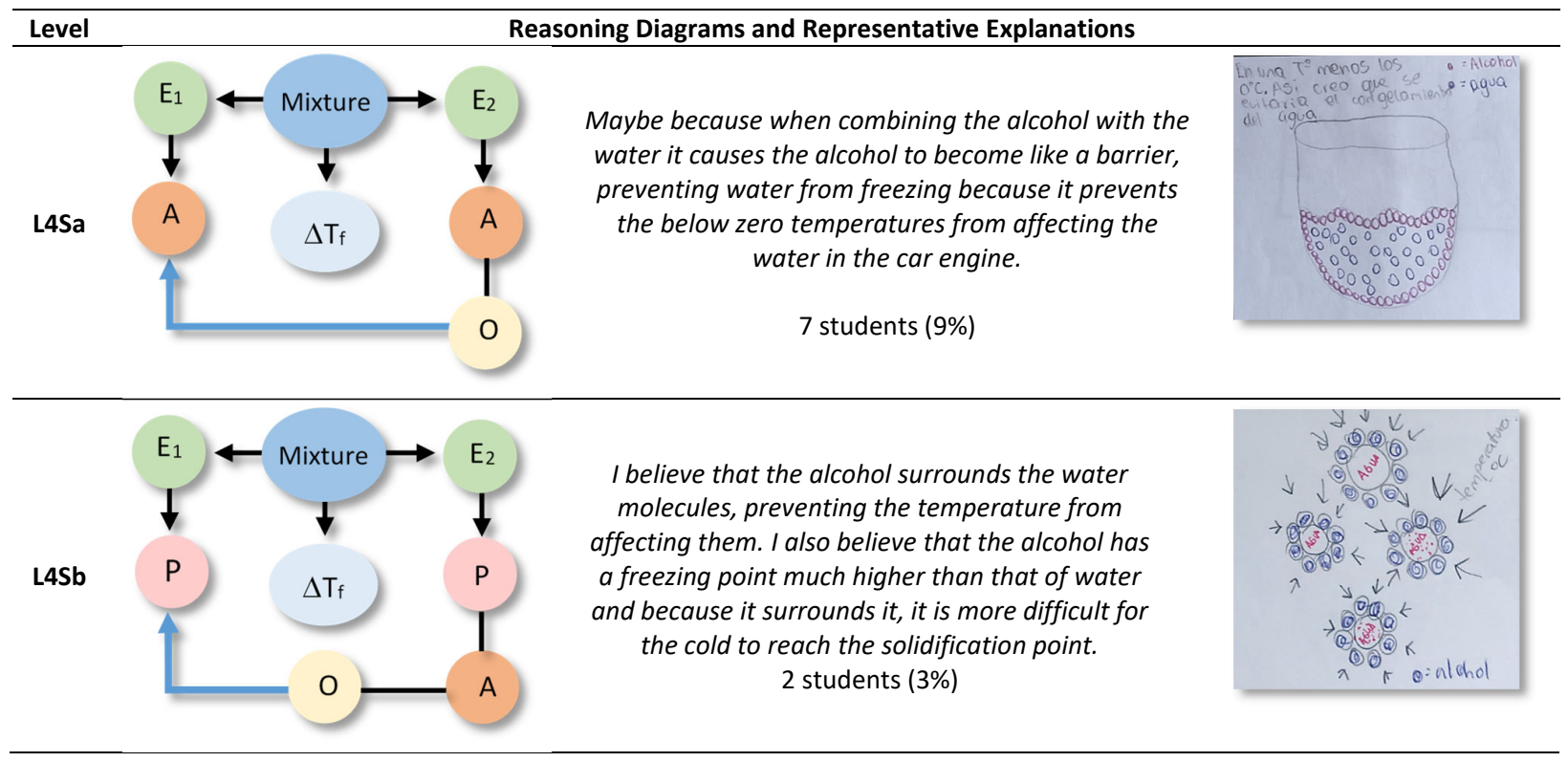

Table 4: Reasoning diagrams and associated representative responses at Level IV. 
- They act as an anti-freezer because the components in the alcohol cause the temperature of water to raise so water doesn't freeze. (L2)

- Because the alcohol generates movement in the water particles causing them not to freeze and then it causes it to evaporate because of the properties of the alcohol. (L3)

c) Alcohol as freezing retardant (12\%): In these cases, study participants tended to think of freezing point depression as involving longer times to freeze. In these cases, it was difficult to determine whether students thought of the antifreeze as only affecting freezing time or implicitly assumed that lower freezing temperatures would be reached in longer times. These types of explanations were observed in explanations at Levels II and IV as illustrated below.

- Pure water freezes faster because there is no solute in it, but in the anti-freezer there is a solute that prevents or delays the freezing of water. (L2)

- The solution takes longer to freeze because it has different components and it takes longer to get ordered to freeze. (L4)

d) Alcohol as an agent that changes water (5\%): In this case, alcohol was seen as an agent that somehow changed the form or composition of water. These types of responses were found in explanations at Levels II and III. For example: - Alcohol is a substance different from water causing it to change its form to defend itself from the cold. (L2)

- The particles of alcohol affect those of water so that water could not freeze, it affects its composition. (L3)

\section{Discussion}

The central goal of our research was to characterise the different types of reasoning manifested by high school chemistry students when building initial written explanations of a natural phenomenon. In particular, our study participants were asked to explain why a mixture of water and alcohol works as an antifreeze. Our analysis revealed that students at the same educational level construct a wide range of explanations for the same phenomenon that are indicative of different reasoning modes ranging from descriptive to relational to simple causal to emerging mechanistic. These modes of reasoning correspond to different levels of sophistication in causal thought and were characterised by paying attention to both the system components (i.e., entities, properties, activities, organisation) and the causal models invoked by the participants in their explanations. Our analytical framework proved to be a productive tool in the identification and characterisation of differences in expressed student reasoning.

The majority of the explanations built by the students in our sample were based on simple associations between system components (relational reasoning), and close to $50 \%$ of the participants thought of alcohol as the active causal agent responsible for the phenomenon. The prevalence of this type of "centralized" view of causality in students' explanations has been highlighted by a variety of authors in previous studies (Grotzer, 2003; Resnick, 1994; Talanquer, 2010). Past studies have also shown that the types of explanations that students build is influenced by the nature of the question they are facing (Gilbert et al., 2000; Talanquer, 2010; Sevian and Talanquer, 2014). Gilbert et al (2000) have proposed six types of explanations based on the task or question to be answered: contextualizing, intentional, descriptive, interpretative, causal, and predictive. In our case, students were expected to generate causal explanations. However, less than half of our study participants actually built them.

A majority of the students in our sample generated non-causal accounts at Levels I (Descriptive) and II (Relational) in our characterisation. Explanations at Level I included simple redescriptions of the targeted phenomenon with or without recognition of the entities present in the system and their relevant properties. Most of these explanations were intentional in nature (Gilbert et al., 2000), focused on highlighting the role of the antifreeze in the car engine. Explanations at Level II recognized relevant entities in the system and some of their properties, establishing either unidirectional or mutual relationships between them. The different types of relationships present in explanations at Level II can be seen as indicators of emerging causality in student reasoning (Grotzer and Perkins, 2000). At this level, students recognized relevant components in a system and linked their presence, or their properties, to the phenomenon under analysis. There was, however, no attempt to build actual causal links between them. Instead, students often applied an additive schema in which the freezing point of the solution was seen as resulting from the average of the properties of the two entities in the mixture. Reliance on an additive schema to explain and make predictions about the properties of chemical systems has been reported previously in the chemistry education research literature (Talanquer, 2008, 2010).

Explanations at Levels III (Simple Causal) and IV (Emerging Mechanistic) involved different forms of relational causality in student reasoning, including simple linear causality and mutual causality (Grotzer and Perkins, 2000). Examples of simple linear causality were observed in explanations at Levels III and IV, while cases of mutual causality were only present in explanations at Level III. Student reasoning in explanations at Level III invoked activities of relevant entities as core components when explaining freezing point depression. In explanations at Level IV (Emerging Mechanistic), the spatio-temporal organisation of alcohol particles played a central role in justifying the observed phenomenon. Although these latter forms of reasoning were mechanistic in nature, they were not aligned with accepted scientific accounts. In non-normative manners, students often attributed central agency to the alcohol (or to the alcohol particles) which was frequently conceived as acting as a barrier or a retardant to the freezing of water, or as a source of energy in the system. Similar forms of causal attribution have been described in past investigations of students' understanding of colligative properties (Çokadar, 2009).

More than half of the students in our sample generated noncausal explanations, which may be indicative of low levels of knowledge integration that would limit their ability to apply scientific knowledge to everyday phenomena (Millar, 2006). Students who were capable of producing causal explanations in many cases referred to causal factors not aligned with accepted scientific accounts. These results are consistent with the findings of national and international standardized assessments that show low levels of performance in Chilean students' ability to explain natural phenomena (ACE 2015).

Although the different types of reasoning represented by increased level of sophistication in Figures 3 and 4 could be thought of as a progression in student learning, we cannot make that claim given the nature of our study. One could assume that the identification of major entities in a system and the recognition of 
their relevant properties are first and necessary steps in the process of learning to explain. The construction of simple associations between entities and properties may follow in the progression, and then move to identification of causal links mediated by interactions between entities and the activities to which they give place. Finally, the identification of spatio-temporal organisations would facilitate the elaboration of mechanistic accounts of the targeted phenomenon. Our findings do not support or contradict this potential learning progression. They just make explicit the range of reasoning types demonstrated by students and illustrate how they can be characterised in a systematic manner.

\section{Implications}

Our analytical approach elicited the wide range of reasoning modes that can be found among a group of students in a single class at the high school level. This diversity highlights one of the major challenges that teachers face in order to advance student thinking in science and chemistry classrooms. Helping all students develop their explanatory abilities is likely to require more individualized assessments, both diagnostic and formative, and instructional interventions. We believe that the framework for analysis introduced in this contribution can be of great use to teachers and researchers in the characterisation of student reasoning. The proposed approach focuses the attention on the identification of specific features in students' explanations and on the types of relationships between them. The representation of these elements in reasoning diagrams simplifies the characterisation of the levels of reasoning that are manifested.

We recognize that different task prompts may trigger responses that require the identification of additional features (e.g., chaining) to characterise expressed reasoning. Nevertheless, a focus on the analysis of the core elements emphasized in our study could help teachers generate inferences about the types of reasoning that students express. Without formal coding, teachers could productively analyse student work by paying attention to a) the specific reasoning features present in students' explanations (i.e., entities, properties, activities, organisation), b) the nature of the relationships between these elements that students highlight, and c) the causal models (e.g., linear, mutual) that they invoke.

Our findings indicate that although the initial explanations generated by many secondary school students may not be very sophisticated in terms of the causal models on which they rely, students at this level are capable of generating mechanistic explanations using particulate models of matter. Teachers should recognize and take advantage of this potential to advance students' explanatory abilities. This demands the creation of multiple opportunities for students to engage in explanation in the classroom, make their thinking visible, share ideas, and receive explicit guidance and feedback on how to build causal mechanistic accounts.

The results of our study suggest that chemistry students' initial explanations of targeted phenomena are likely to be nonnormative, relying on intuitive assumptions about the properties and behaviours of chemical substances and processes (Talanquer, 2006, 2013; Taber and García-Franco, 2010). From this perspective, one could have expected that a phenomenon like freezing point depression may be attributed to the actions of an agent that actively hinders the freezing process. Teachers who want to develop their students' explanatory abilities should accept these types of non-canonical initial explanations rather than suppress them as soon as they are expressed. It is important for teachers to be responsive to alternative ideas, pressing students to further develop their thinking through causal mechanistic reasoning. Once different models are built in the classroom based on a set of diverse ideas, they can be compared and contrasted to evaluate their scope and limitations. It is in this evaluation process that teachers can more effectively intervene to introduce or move student thinking closer to the scientific accounts.

Advancing student thinking about colligative properties such as freezing point depression requires the construction of mechanisms that recognize the probabilistic nature of change at the particulate level. Students should be engaged in the analysis and discussion of how dynamic equilibrium is established in systems where competing processes take place (e.g., melting and solidification). Once this understanding is developed, inferences about the effects of introducing perturbations in those equilibria (e.g., adding a solute) should be easier to generate. Existing research shows that from a very young age, students possess cognitive resources that allow them to make sense of emergent phenomena and differentiate them from centralized (or directed) processes (Grotzer et al., 2017).

\section{Limitations}

Our qualitative study focused on the analysis of the written explanations generated by a single group of $10^{\text {th }}$ grade students working on a single task in a particular topic in the Chilean chemistry curriculum. Consequently, one should be careful in generalizing the scope of our findings. More investigations are needed to evaluate whether our analytical framework is robust and productive in the analysis and characterisation of the reasoning manifested by diverse types of students when asked to build causal explanations for a wide range of phenomena in different contexts. These types of studies could be strengthened by conducting individual interviews to gain more in-depth insights into student reasoning.

\section{Conflicts of interest}

There are no conflicts to declare.

\section{Acknowledgements}

This work was supported by CONICYT-21161280, which provided a scholarship for the first author of this paper, and by the research project FONDECYT 1160148 in Chile.

\section{References}

Agencia de Calidad de la Educación [ACE], (2015): Programa para evaluación internacional de estudiantes OCDE, Santiago, Chile.

Akaygun S. and Jones L. L., (2014), Words or Pictures: A comparison of written and pictorial explanations of physical and chemical equilibria. Int. J. Sci. Educ. 36(5), 783-807.

Assaraf O. B. Z. and Orion N., (2005), Development of system thinking skills in the context of earth system education. J. Res. Sci. Teach., 42(5), 518-560.2.

Becker N., Noyes K., and Cooper M., (2016), Characterizing students' mechanistic reasoning about London dispersion forces. J. Chem. Educ., 93(10), 1713-1724.

Bhattacharyya G., (2013), From source to sink: Mechanistic reasoning using the electron-pushing formalism. J. Chem. Educ., 90(10), 1282-1289.

Biggs J. and Collis K., (1982), Origin and description of the SOLO taxonomy. Eval. Qual. Learn., 1, 17-31.

Braaten M. and Windschitl M., (2011), Working toward a stronger conceptualization of scientific explanation for science education. Sci. Educ., 95(4), 339-370.

Brewer W. F., Chinn C. A., and Samarapungavan A., (1998), 
Explanation in scientists and children. Minds Mach., 8, 119136.

Brown N. J. S., Nagashima S. O., Fu A., Timms M., and Wilson M., (2010), A framework for analyzing scientific reasoning in assessments. Educ. Assess., 15(3), 142-174.

Bybee R., McCrae B., and Laurie R., (2009), PISA 2006: An assessment of scientific literacy. J. Res. Sci. Teach., 46(8), 865-883.

Cabello V. M., (2017), Role-playing for learning to explain scientific concepts in teacher education. J. Sci. Educ., 18(2), 67-70.

Christidou V. and Hatzinikita V., (2006), Preschool children's explanations of plant growth and rain formation: $A$ comparative analysis. Res. Sci. Educ., 36(3), 187-210.

Çokadar H., (2009), First year prospective teachers ' perceptions of molecular polarity and properties of solutions. Asian J. Chem., 21(1), 75-85.

Creswell J. W., (2013), Qualitative inquiry and research design: choosing among five approaches, Lincoln: Sage.

Gilbert J. K., Boulter C. J., and Rutherford M., (2000), Explanations with Models in Science Education. in Developing Models in Science Education. Dordrecht: Springer Netherlands, pp. 193208.

Grotzer T. A., (2003), Learning to understand the forms of causality implicit in scientifically accepted explanations. Stud. Sci. Educ., 39(1), 74.

Grotzer T. A. and Perkins D. N., (2000), A Taxonomy of Causal Models : The Conceptual Leaps Between Models and Students ' Reflections on Them. in National Association of Research in Science Teaching (NARST). New Orleans, LA.

Grotzer T. A., Derbiszewska K., and Solis S. L., (2017), Leveraging fourth and sixth graders' experiences to reveal understanding of the forms and features of distributed causality. Cogn. Instr. 35(1), 55-87.

Hempel C. G. and Oppenheim P., (1948), Studies in the logic of explanation. Philos. Sci., 15(2), 135-175.

Izquierdo M. and Aliberas J., (2004), Pensar, actuar i parlar a la classe de ciències. Per un ensenyament de les ciències racional i razonable, Cerdanyola: Servei Publicacions UAB.

Machamer P., Darden L., and Craver C., (2000), Thinking about Mechanisms. Philos. Sci., 67(1), 1-25.

Miles; M. B.; \& Huberman; A. M., (1994), Qualitative data analysis: an expanded sourcebook, 2nd ed. Thousand Oaks; CA: Sage.

Millar R., (2006), Twenty First Century Science: Insights from the design and implementation of a scientific literacy approach in school science. Int. J. Sci. Educ., 28(13), 1499-1521.

National Research Council [NRC], (2013), A framework for $\mathrm{K}-12$ science education, Washington, D.C.: National Academies Press.

Perkins D. N. and Grotzer T. A., (2005), Dimensions of causal understanding: The role of complex causal models in students' understanding of science. Stud. Sci. Educ., 41(1), 117-166.

Resnick M., (1994), Turtles, termites, and traffic jams : explorations in massively parallel microworlds, MIT Press.

Rottman B. M. and Keil F. C., (2011), What matters in scientific explanations: Effects of elaboration and content. Cognition, 121(3), 324-337.

Russ R. S., Coffey J. E., Hammer D., and Hutchison P., (2009), Making classroom assessment more accountable to scientific reasoning: A case for attending to mechanistic thinking. Sci. Educ., 93(5), 875-891.

Russ R. S., Scherr R. E., Hammer D., and Mikeska J., (2008),
Recognizing mechanistic reasoning in student scientific inquiry: A framework for discourse analysis developed from philosophy of science. Sci. Educ., 92(3), 499-525.

Ryder J., (2001), Identifying science understanding for functional scientific literacy. Stud. Sci. Educ., 36(1), 1-44.

Salmon W. C. [1984], Scientific explanation and the causal structure of the world, Princeton: Princeton University Press

Sandín M. P., (2003), Investigación cualitativa en educación: fundamentos y tradiciones, Madrid, España: Mc Graw Hill.

Sevian H. and Talanquer V., (2014), Rethinking chemistry: a learning progression on chemical thinking. Chem. Educ. Res. Pract., 15(1), 10-23.

Southard K. M., Espindola M. R., Zaepfel S. D., and Bolger M. S., (2017), Generative mechanistic explanation building in undergraduate molecular and cellular biology*. Int. J. Sci. Educ., 39(13), 1795-1829.

Taber K. S. and García-Franco A., (2010), Learning processes in chemistry: Drawing upon cognitive resources to learn about the particulate structure of matter. J. Learn. Sci., 19(1), 99142.

Talanquer V., (2006), Commonsense chemistry: A model for understanding students' alternative conceptions. J. Chem. Educ., 83(5), 811-816.

Talanquer V., (2007), Explanations and teleology in chemistry education. Int. J. Sci. Educ., 29(7), 853-870.

Talanquer V., (2008), Students' predictions about the sensory properties of chemical compounds: Additive versus emergent frameworks. Sci. Educ., 92(1), 96-114.

Talanquer V., (2010), Exploring dominant types of explanations built by general chemistry students. Int. J. Sci. Educ., 32(18), 23932412.

Talanquer V., (2013), How do students reason about chemical substances and reactions? In G. Tsaparlis and H. Sevian (Eds.), Concepts of matter in science education. Series Innovations in Science and Technology Education, Vol. 19, Dordrecht: Springer, pp. 331-346.

Talanquer V., (2018), Exploring mechanistic reasoning in chemistry. In J. Yeo, T. W. Teo, \& K.S. Tang (Eds.) Science Education Research and Practice in Asia-Pacific and Beyond, Singapore: Springer, pp. 39-52.

Tamir P., (1991), Assessing students' difficulties in causal reasoning in biology-a diagnostic instrument. J. Biol. Educ., 25, 302-307.

Tang K. S., (2016), Constructing scientific explanations through premise-reasoning-outcome (PRO): an exploratory study to scaffold students in structuring written explanations. Int. J. Sci. Educ., 38(9), 1415-1440.

van Mil M. H. W., Boerwinkel D. J., and Waarlo A. J., (2013), Modelling molecular mechanisms: A framework of scientific reasoning to construct molecular-level explanations for cellular behaviour. Sci. Educ., 22(1), 93-118.

Weinrich M. L. and Talanquer V., (2016), Mapping students' modes of reasoning when thinking about chemical reactions used to make a desired product. Chem. Educ. Res. Pract., 394(17), 394-406.

Windschitl M., Thompson J. J., and Braaten M. L., (2018), Ambitious science teaching, Cambridge, MA: Harvard Education Press.

Yeo J. and Gilbert J. K., (2014), Constructing a scientific explanation-A narrative account. Int. J. Sci. Educ., 36(11), 1902-1935. 